\title{
Modeling the effectiveness of olfactory testing to limit SARS-CoV-2 transmission
}

\author{
Daniel B. Larremore ${ }^{1,2}$, Derek Toomre ${ }^{3}$, and Roy Parker ${ }^{2,4,5}$ \\ ${ }^{1}$ Department of Computer Science, University of Colorado Boulder \\ ${ }^{2}$ BioFrontiers Institute, University of Colorado Boulder \\ ${ }^{3}$ Department of Cell Biology, Yale University School of Medicine \\ ${ }^{4}$ Department of Biochemistry, University of Colorado Boulder \\ ${ }^{5}$ Howard Hughes Medical Institute
}

\begin{abstract}
A central problem in the COVID-19 pandemic is that there is not enough testing to prevent infectious spread of SARS-CoV-2, causing surges and lockdowns with human and economic toll. Molecular tests that detect viral RNAs or antigens will be unable to rise to this challenge unless testing capacity increases by at least an order of magnitude while decreasing turnaround times. Here, we evaluate an alternative strategy based on the monitoring of olfactory dysfunction, a symptom identified in $76-83 \%$ of SARSCoV-2 infections-including those with no other symptoms - when a standardized olfaction test is used. We model how screening for olfactory dysfunction, with reflexive molecular tests, could be beneficial in reducing community spread of SARS-CoV-2 by varying testing frequency and the prevalence, duration, and onset time of olfactory dysfunction. We find that monitoring olfactory dysfunction could reduce spread via regular screening, and could reduce risk when used at point-of-entry for single-day events. In light of these estimated impacts, and because olfactory tests can be mass produced at low cost and self-administered, we suggest that screening for olfactory dysfunction could be a high impact and costeffective method for broad COVID-19 screening and surveillance.
\end{abstract}

To whom correspondence should be addressed:

daniel.larremore@colorado.edu,derek.toomre@yale.edu,roy.parker@colorado.edu 


\section{Introduction}

The COVID-19 pandemic has created a global public health crisis. Due to the fact that SARS-CoV-2 can spread from individuals with and without overt symptoms [1-3], there remains an urgent need to identify infected individuals prior to onward spread. To meet this need, large efforts are currently underway to develop, regulate, and mass produce rapid, inexpensive, and effective screening tests for viral antigens that could be used repeatedly and at wide scale. However, an alternative approach is to utilize widespread screening of symptoms of SARS-CoV-2 infection, which could then stratify individuals with symptoms for follow-up molecular testing.

Fever was advanced early in the pandemic as a potential screening symptom but failed [4] because fever $\left(\geq 38^{\circ} \mathrm{C}\right.$ ) is (i) only present in 18-26\% of COVID-19 cases [5-7], (ii) occurs in many diseases (e.g. Flu) and is not specific to COVID-19 [8], and (iii) lasts only 1.5 days on average [5]. Nevertheless, temperature checks persist at many hospital entrances due to the speed and convenience of contactless thermometers and thermography, and because low cost yet effective alternatives are not widely available.

In contrast to fever, three key observations supported by a large and growing body of evidence indicate that olfactory dysfunction-hyposmia or anosmia — may be a superior screening and surveillance symptom. We briefly review this evidence, which may also be found in Supplementary Table S1. First, olfactory dysfunction is the best predictor symptom of COVID-19. Studies from both the U.S. CDC [9] and large cross-sectional questionnaires of thousands of subjects $[10,11]$ have shown that OD predicts COVID-19 with an odds ratio of 10.4, a 4-10-fold higher association with COVID-19 than fever [9]. While OD is only weakly associated with influenza ${ }^{1}$, it can be caused by other viral infections, head trauma, and chronic dementia [12].

Second, OD occurs in 76-83\% of COVID-19 infections when measured with a quantitative olfactory test, such as a smell identification or threshold test [13-15]. Although reported prevalence of OD associated with PCR confirmed SARS-CoV-2 infection has varied widely in individual studies, pooled meta-analyses have established symptom prevalence estimates of $41 \%$ ( $n=24$ studies [13]), 44\% ( $n=34$ studies [14]), and 53\% ( $n=19$ studies [15]) when OD was self reported. Strikingly, when OD was measured with a quantitative olfactory test, its estimated prevalence in meta-analyses increased to 83\% [13], 77\% [14], and $76 \%$ [15]. The stage or severity of disease may also play a role in the prevalence of OD. Several reports indicate that patients with mild COVID-19 had a high incidence of OD, ranging from $68 \%$ to $86 \%$, but rates were far lower among severely ill patients $[10,16]$. However, this may be due to the fact that hospitalized patients are typically tested later in the course of infection, at which point OD symptoms may have largely resolved [17, 18]. Furthermore, new reports using objective olfactory tests have found that $79 \%$ (22 of 28) of otherwise asymptomatic adults [19] and $86 \%$ of symptomatic children [11] showed partial loss of smell (hyposmia). To capture the entire range of prevalence estimates, the present study considers the implications of a range of COVID-19-associated OD prevalence values, ranging from $25 \%$ to $90 \%$.

Third, onset of olfactory dysfunction may precede overt symptoms (e.g. difficulty breathing, cough, and diarrhea) by days [20-27], and COVID-19-induced olfactory dysfunction has been shown to last roughly 7 days in clinical studies [10,13,15,21], although in some cases may last for months [28]. Combined, olfactory dysfunction's prevalence, specificity, onset time, and duration have led us to hypothesize that, while it is not an overt symptom of COVID-19 and is underreported in self-reporting surveys [13, 15], standardized olfactory dysfunction testing may be a valuable but underutilized screening and surveillance

\footnotetext{
${ }^{1}$ https://www.cdc.gov/flu/symptoms/flu-vs-covid19.htm
} 
tool.

Recent modeling work has shown that, for COVID-19 screening via repeated molecular testing, test frequency and turnaround time are critical, while test sensitivity is secondary [29, 30]. Standardized olfactory dysfunction testing may be sufficiently low cost to be performed frequently, and olfactory dysfunction testing can be self-administered in minutes without personal protective equipment (PPE). We therefore considered whether olfactory dysfunction could be effectively used in a similar repeated regimen to proposed molecular testing [29, 31], and to what extent its effectiveness depends on its onset time, duration, and prevalence among those who are infected but do not experience overt COVID-19 symptoms. In this work, we define olfactory dysfunction to include any defect in olfaction that could be detected with a simple quantitative olfaction test, including the complete loss of smell (anosmia) and partial loss of smell (hyposmia).

\section{Methods summary}

We analyzed how screening for olfactory dysfunction could impact COVID-19 spread while varying the prevalence of olfactory dysfunction among infected individuals, its duration, the timing of onset, and the frequency of testing. In each case we analyzed the impact of screening regimens in two manners.

In one set of analyses, we used a simple fully-mixed Susceptible-Exposed-Infectious-Recovered (SEIR) model representing a population of 20,000 people, similar to a large university setting, with a constant rate of external infection approximately equal to one new import per day [29]. Individual viral loads were simulated for each infection based on key features of latency, proliferation, peak, and clearance identified in the literature (Materials and Methods; [29, 32]). Infected individuals who scored positive for olfactory dysfunction were considered to be tested for SARS-CoV-2 by RT-PCR and were isolated if positive. To better model viral dynamics and behavior in the presence of overt and noticeable symptoms independent of olfactory dysfunction, 35\% of modeled individuals had viral load trajectories with prolonged clearance times [32,33], and were modeled to self isolate within 0-2 days of peak viral load, independent of screeningrelated testing. Contact tracing was not included to more conservatively estimate the impacts of screening alone $[34,35]$. We used a value of $R_{0}$ of 1.5 , to reflect accelerating but partially mitigated transmission.

In a second set of analyses, we simulated the viral loads, possible onset of olfactory dysfunction, and infectiousness curves, of 10,000 individuals and then examined how much infectiousness was removed from the population by different olfaction testing regimens. This allowed us to examine the results of modeling under a range of different screening strategies and olfactory dysfunction parameters by estimating the impact, in each case, on the reproductive number $R$.

In all analyses, we considered $80 \%$ of the population to participate in the screening protocol, examined performing olfactory testing either daily, every third day, or weekly, and infectiousness was taken to be proportional to the logarithm of viral load in excess of $10^{6}$ virions $/ \mathrm{ml}$ [29].

One issue arising when screening for olfactory dysfunction is that defects in olfaction may be caused by other respiratory illness or early-stage neurodegenerative diseases [36, 37]. COVID-19-independent olfactory dysfunction has been estimated to affect $3-25 \%$ of the general population, with the largest increase among older adults [36]. Higher specificity for COVID-19 olfactory dysfunction is possible, with some loss of sensitivity, by using test criteria that select for anosmia and severe hyposmia, excluding mild hyposmia and individuals with a stuffy or runny nose- a common symptom in other viral infections, but rare (2-4\%) in COVID-19[38]. In consideration of this issue, we modeled that $20 \%$ of individuals would have a COVID-19- 

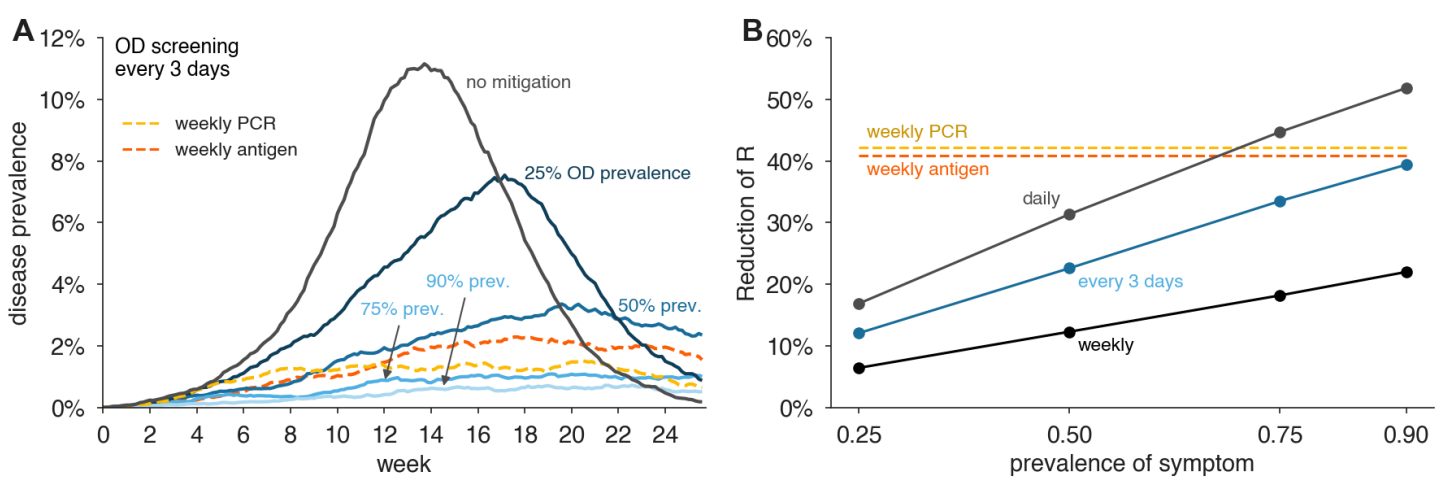

Figure 1: Impact of olfactory dysfunction prevalence on its effectiveness to limit viral spread. (A) Examples of viral spread in a community of 20,000 individuals performing olfactory dysfunction (OD) screening every three days. No mitigation (black), Prevalence of symptom shown are: 25\% (dark blue), 50\% (medium blue), 75\% (light blue), $90 \%$ (lightest blue). In this analysis, olfactory dysfunction is modeled to last 7 days and begin two days after viral levels reach $\sim 1000$ virions/ml. (B) Reduction of reproductive number $R$ with different testing regimens showing the impact of symptom prevalence with weekly (black line), every three days (blue line), or daily (gray line), testing for olfactory dysfunction. For comparison, weekly RT-PCR testing with a one day turnaround (dashed yellow line) and weekly antigen testing (dashed red line) are shown. We consider 80\% participation in testing and 20\% COVID-19independent olfactory dysfunction in both panels.

independent olfactory dysfunction, allowing us to examine the value of olfaction screening under near-worst case real-world conditions [36, 39, 40]. Taken together, all simulations that follow assume that only $64 \%$ ( $80 \%$ of $80 \%$ ) of individuals are both willing and able to participate in an olfactory screening program. Details of both models and parameters are fully described in Materials and Methods.

\section{Results}

\section{Olfactory dysfunction screening can impact population spread}

We first examined how the prevalence of olfactory dysfunction during COVID-19 infection would impact its use in a repeated screening regimen. Olfactory dysfunction has been suggested to occur in $50-90 \%$ of COVID-19 infections for roughly one week [10, 13, 15, 21]. Thus, we modeled olfactory dysfunction as a symptom able to be detected with repetitive olfactory testing in 25\% (underestimate), $50 \%$ (low estimate), $75 \%$ (realistic), and $90 \%$ (high estimate) of infected individuals with an olfactory dysfunction duration of 7 days [10,21]. Although reports indicate that olfactory dysfunction is an early COVID-19 symptom [2027], its precise timing relative to viral loads is unclear. Given this uncertainty, we initially modeled the average onset of olfactory dysfunction as occurring two days after viral loads reached detectable levels based on RT-PCR, consistent with studies indicating that onset of olfactory dysfunction precedes onset of overt symptoms [20-27].

We observed that screening for olfactory dysfunction daily (Fig. S1A) or every third day (Fig. 1A) limited viral spread in simulations, provided symptom prevalence was greater than $50 \%$. Notably, when symptom prevalence was $75 \%$ or higher olfactory screening every third day was comparable in effectiveness to weekly RT-PCR testing with a one day turnaround time (Fig. 1; yellow dashed line) or weekly antigen testing (Fig. 1; red dashed line). At 50\% symptom prevalence, viral spread was partially controlled by testing every three days (Fig. 1), and more effectively controlled with daily testing (Fig. S1A). Weekly olfactory 

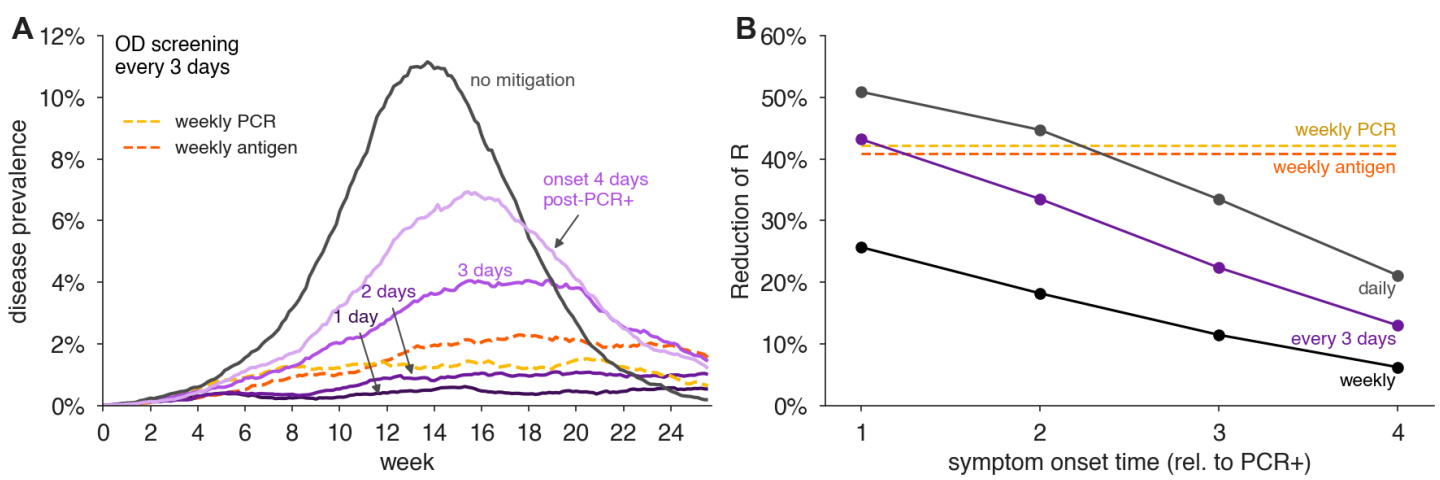

Figure 2: Impact of the timing of olfactory dysfunction onset on its effectiveness to limit viral spread. (A) Examples of viral spread in fully-mixed community of 20,000 individuals performing olfactory dysfunction (OD) screening every three days. olfactory dysfunction is modeled to be present in $75 \%$ of infected individuals, and to last 7 days. Timing of olfactory dysfunction is varied from one to four days after viral loads reach $\sim 1000$ virions $/ \mathrm{ml}$ (purple shaded lines as indicated). No mitigation is shown as black line. (B) Reduction of reproductive number $R$ with different testing regimens showing the impact of timing of symptom onset with weekly (black line), every three days (purple line), or daily (gray line), testing for olfactory dysfunction. For comparison, weekly RT-PCR testing with a one day turnaround (dashed yellow line) and weekly antigen testing (dashed red line) are shown. We consider $80 \%$ participation in testing and 20\% COVID-19-independent olfactory dysfunction in both panels.

screening mitigated but failed to fully control outbreaks except when symptom prevalence was modeled at 90\% (Fig. S1B).

By estimating the reduction in the reproductive number $R$ for each scenario, we were able to perform direct comparisons of the predicted effectiveness of screening strategies across transmission scenarios (Fig. 1B). This illustrates how daily olfactory testing when symptom prevalence is $75 \%$ or greater is predicted to be slightly more effective than weekly antigen testing or RT-PCR with a one day turnaround time.

\section{Impact of timing of olfactory dysfunction onset}

Although olfactory dysfunction is an early symptom of COVID-19 [20-24, 26], there are limited data on the exact timing of symptom onset and its variability between individuals. Given this uncertainty, we performed additional analyses on how olfactory screening would be affected by the timing of symptom onset (Fig. 2). Assuming $80 \%$ participation in testing and $20 \%$ of the population having COVID-19-independent olfactory dysfunction, we considered the central estimates of $75 \%$ symptom prevalence and seven days of duration, and varied the timing of symptom onset from one to four days after viral loads are detectable by RT-PCR.

We observed that daily, or every three days, olfactory testing was sufficient to keep viral infections from developing into an outbreak, provided that olfactory dysfunction typically occurs within two days of positivity by RT-PCR (Fig. 2). However, when symptom onset was three days after detectable viral loads, epidemic growth was poorly controlled with testing every three days, but could be controlled with daily testing (Figs. 2A and S2A). By estimating the impacts of screening regimens on the reproductive number, we found comparable impact on transmission between weekly PCR and olfactory screening every 3 days (for onset two days after PCR positivity) or daily (for onset three days after PCR positivity). Even when symptom onset was four days after PCR positivity, viral spread was reduced by daily olfactory testing (Fig. S2A). Weekly testing was largely ineffective when symptom onset was later than one day after viral loads reached detectable levels (Fig. S2B). 

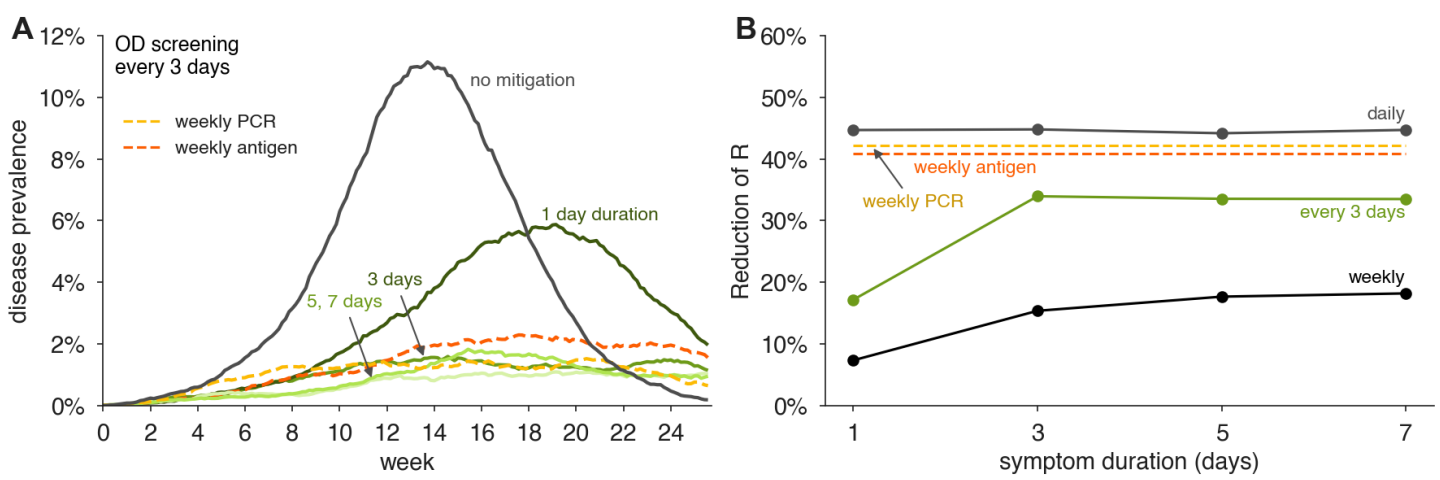

Figure 3: Impact of duration of olfactory dysfunction on its effectiveness to limit viral spread. (A) Examples of viral spread in fully-mixed community of 20,000 individuals performing olfactory dysfunction (OD) screening every three days. Olfactory dysfunction is modeled to be present in $75 \%$ of infected individuals, and to begin two days after viral levels reach $\sim 1000$ virions/ml. Duration of olfactory dysfunction is varied from 7 days (lightest green), 5 days (light green), 3 days (green), to 1 day (dark green). No mitigation is shown as black line. (B) Reduction of reproductive number $R$ with different testing regimens showing the impact of olfactory dysfunction duration with weekly (black line), every three days (green line), or daily (gray line), testing for olfactory dysfunction. For comparison, weekly RTPCR testing with a one day turnaround (dashed yellow line) and weekly antigen testing (dashed red line) are shown. We consider $80 \%$ participation in testing and 20\% COVID-19-independent olfactory dysfunction in both panels.

\section{Impact of olfactory dysfunction duration}

Additional analyses argue that olfactory dysfunction screening could be an effective COVID-19 control mechanism even if the duration of olfactory dysfunction is shorter. For these analyses, we considered the case of $80 \%$ participation, $20 \%$ of the population having COVID-19-independent olfactory dysfunction, $75 \%$ symptom prevalence, symptom onset two days after viral loads become detectable by RT-PCR, and varied the symptom duration from one, three, five, or seven days.

We observed that testing every third day for olfaction controlled epidemic growth when the duration of olfactory dysfunction was three to seven days (Fig. 3). Even if olfactory dysfunction lasted only one dayunlikely based on current observations-daily testing would nevertheless allow effective control. Weekly testing for olfaction was insufficient to maintain viral control (Figs. 3B, S3B, and S4L). To investigate interactions between onset timing and duration, we systematically modeled combinations of each, finding that testing for olfactory dysfunction every three days was effective, as assessed either by reductions in $R$ (Fig. 4) or by viral containment in community simulations (Fig. S4), provided that olfactory dysfunction lasted at least three days, and symptom onset was within 2 days of viral loads detectable by RT-PCR. Importantly, daily olfactory testing was sufficient to control viral spread even when the olfactory dysfunction onset was three days after detectable viral levels and lasted only for a single day (Fig. S4A).

These analyses demonstrate that screening for olfactory dysfunction can be an effective control mechanism, even if average symptom duration is short, and symptom onset occurs within three days of detectable viral loads. More frequent testing for olfaction is required for effective control when the duration of olfactory dysfunction is short, and/or symptom onset is later in viral infection, with many scenarios providing reductions in the reproductive number equivalent to, or better than, weekly RT-PCR or antigen testing (Fig. 4). 

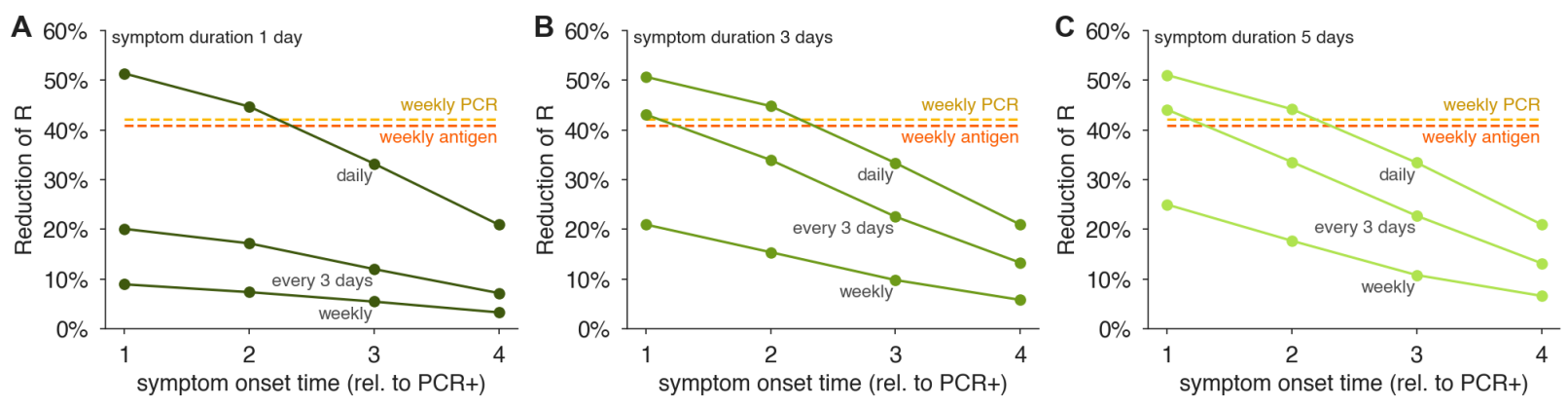

Figure 4: Impact of the timing of onset and duration of olfactory dysfunction on its effectiveness to limit viral spread. Reductions of the reproductive number $R$ by testing daily, every third day, or weekly for olfaction dysfunction are shown for olfactory dysfunction lasting (A) one day, (B) three days, and (C) five days, for varying symptom onset times, relative to when viral levels reach $\sim 1000$ virions $/ \mathrm{ml}$. We consider $80 \%$ participation in testing and $75 \%$ prevalence of olfactory dysfunction as a COVID-19 symptom, and 20\% COVID-19-independent olfactory dysfunction in all panels.

\section{Screening for olfactory dysfunction to mitigate an outbreak.}

To investigate whether olfactory screening could be effective at controlling an ongoing outbreak, we simulated epidemics in the fully-mixed SEIR model, such that a screening regimen began only when prevalence reached $2 \%$ of the population. We used the central estimates of $75 \%$ olfactory dysfunction prevalence, symptom onset two days after detectable viral levels, and a symptom duration of 7 days. We again considered $80 \%$ participation in testing, with a further $20 \%$ of the population having COVID-19-independent olfactory dysfunction. We observed that screening daily or every third day was sufficient to bring the outbreak under control (Fig. 5), and under these conditions, would be similar to weekly RT-PCR or antigen testing.

\section{Olfactory dysfunction screening regimens are cost-effective}

To estimate the costs of modeled interventions, in each simulation we tracked the number of required olfactory dysfunction tests and follow up RT-PCR assays over a 120-day simulation (Table 1). In the repeated testing cases, we assumed individuals with COVID-19-independent olfactory dysfunction would not be tested by RT-PCR, and therefore the estimated costs of RT-PCR are minimum estimates. We found that a mitigation strategy based on weekly RT-PCR assays with $80 \%$ participation would reduce $R$ by $42 \%$ but cost $\$ 5.3$ million in tests, even at the low rate of $\$ 20$ per RT-PCR test (\$100 is more typical). A similar weekly screening program using $\$ 10$ antigen tests would reduce $R$ by $41 \%$ and cost $\$ 2.7$ million. However, mitigation based on olfactory screening every three days with follow-up RT-PCR would cost $\$ 130,000$ in tests, assuming $\$ 0.25$ per olfactory test, and reduce $R$ by (34\%; see Table 1$)$. By comparing the cost per percentage point reduction in $R$, olfactory screening was $16 \times$ or $31 \times$ more effective than antigen or PCR screening, respectively. Cost estimates do not include costs of staffing, PPE, or sample collection and transport which may be required for testing via RT-PCR.

\section{Olfactory dysfunction as a point of entry screening tool}

Due to the immediate results of currently available olfactory dysfunction tests, we also examined the conditions under which they would be useful as point-of-entry screening tools, analogous to screening of airline passengers or individuals entering a social event with rapid antigen tests. We observed that screening for olfactory dysfunction removed same-day infectiousness in proportion to the fraction of individuals showing 


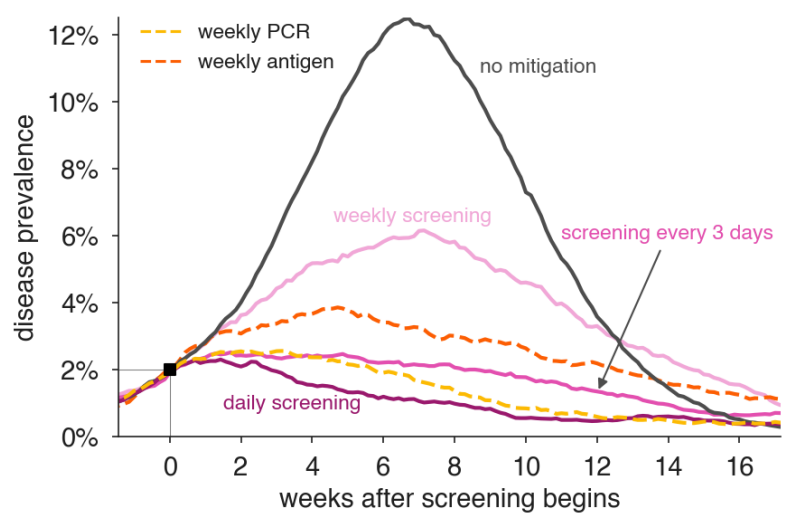

Figure 5: Impact of olfactory dysfunction screening on an ongoing viral outbreak Examples of the impact of screening programs based on olfactory dysfunction on controlling an ongoing outbreak. Viral spread in a fully-mixed community of 20,000 individuals was allowed to proceed until prevalence reached $2 \%$, at which point olfactory dysfunction (OD) screening was initiated either daily (dark red), every three days (red), or weekly (pink). For comparison, weekly RT-PCR testing with a one day turnaround (dashed yellow line) and weekly antigen testing (dashed red line) are shown. Olfactory dysfunction is modeled to be present in $75 \%$ of infected individuals, last 7 days, and to begin two days after viral levels reach $\sim 1000$ virions $/ \mathrm{ml}$. Our modeling assumed that $80 \%$ of people would opt to participate in testing and that $20 \%$ of people would be unable to participate due to COVID-19-independent olfactory dysfunction. Scenario without mitigation is shown for comparison (black).

\begin{tabular}{|l|c|r|r|r|r|}
\hline screening regimen & OD tests & PCR/Ag tests & cost (millions) & reduction in $R$ & cost effectiveness \\
\hline \hline OD daily & $1,531,351$ & 151 & $\$ 0.39$ & $44.7 \%$ & 115.9 \\
\hline OD every 3 days & 509,360 & 314 & $\$ 0.13$ & $33.5 \%$ & 250.7 \\
\hline OD weekly & 213,963 & 457 & $\$ 0.06$ & $18.2 \%$ & 290.7 \\
\hline PCR every 3 days & - & 633,565 & $\$ 12.67$ & $68.4 \%$ & 5.4 \\
\hline PCR weekly & - & 265,695 & $\$ 5.31$ & $42.1 \%$ & 7.9 \\
\hline Ag every 3 days & - & 632,914 & $\$ 6.33$ & $67.6 \%$ & 10.7 \\
\hline Ag weekly & - & 268,088 & $\$ 2.68$ & $40.9 \%$ & 15.2 \\
\hline
\end{tabular}

Table 1: Cost effectiveness of repeated screening programs. Numbers of olfactory dysfunction (OD) tests and PCR tests ordered are translated to total costs for a 120 day testing program at a rate of $\$ 0.25$ per OD test, $\$ 10$ per antigen (Ag) test, and $\$ 20$ per PCR test in simulations of a population of 20,000 individuals with $R_{0}=1.5$, Cost effectiveness is calculated as the percentage reduction in $R$ per million dollars. Individuals positive for olfactory dysfunction received a confirmatory PCR test. Olfactory dysfunction is modeled to be present in $75 \%$ of infected individuals, last 7 days, and to begin two days after viral loads reach 1000 virions $/ \mathrm{ml}$, with $20 \%$ of individuals experiencing COVID-19-independent olfactory dysfunction. We consider $80 \%$ participation in testing of any type. 
that symptom, and was more effective the earlier symptom onset occurred (Fig. S5A). Symptom screening is $\sim 80 \%$ effective when $90 \%$ of the infected individuals show the symptom (Fig. S5A). Notably, even with only $50 \%$ of infected individuals showing olfactory dysfunction over $40 \%$ of infectiousness was removed provided symptom onset occurred no later than two days after sufficient viral load to be detected by RT-PCR testing (Fig. S5A). In contrast, a symptom similar to fever, which is short lived and seen in a small number of infected individuals [5-7], removes at most 20\% of infectiousness (Fig. S5A).

One limitation of using olfactory dysfunction as a single step screen for COVID-19 is the prevalence of non-COVID-19 related olfactory dysfunction, often estimated at 3-5\% of the general population [36, 39, 40], but with higher rates among particular groups, including older adults [36]. To address this issue, we considered a two-step screening procedure in which those failing an olfaction test would be given a reflex rapid antigen test, allowing those with COVID-19-independent olfactory dysfuction to enter the event. This two-step screening procedure was as effective as the one-step olfaction-only procedure, was cost effective ( $\sim \$ 0.75$ to $\$ 2.33$ per person), and reduced false positives that were inappropriately denied entry compared to either test alone. The rapid turnaround time of both olfactory dysfunction testing and rapid antigen testing, suggests that this two-step approach would be effective and superior to an olfaction-only screening procedure (Fig. S5B,C).

\section{Discussion}

An important contribution of this modeling is to demonstrate that monitoring of olfactory dysfunction could be effective at controlling the spread of SARS-CoV-2. Our analysis, and data in the literature, argue that olfactory dysfunction during COVID-19 meets all the necessary criteria of prevalence and specificity, timing, and duration for being effective in pandemic control. First, our modeling showed that the estimated 75\% prevalence of olfactory dysfunction among those with and without other overt COVID-19 symptoms [13, 15, 19] was sufficiently high to have a substantial impact in screening regimens (Fig. 1). Second, we showed that screening would be effective even if onset of olfactory dysfunction is 2 days after detectable levels of virus by RT-PCR, with increasing predicted effectiveness with earlier onset times (Fig. 2). To date, there have been few prospective studies of loss of smell in COVID-19 patients, but existing evidence indicates an earlier onset of olfactory dysfunction than overt symptom like fever and headache [20-23,26]. Determination of the average timing of olfactory dysfunction relative to viral load in COVID-19 patients will be critical to implementing effective screening protocols. Third, our analysis suggests the even if the duration of olfactory dysfunction is as short as one day (the exact duration and its variability in infected individuals with no other overt symptoms is unknown), frequent monitoring for olfaction can still be effective for epidemic control (Fig. 3). Our simulations suggest effective epidemic mitigation can be achieved under all conditions with daily testing; with longer durations and earlier symptom onset, epidemics can be controlled with testing every three days.

Olfactory screening is inexpensive and scalable, two critical factors for large population-scale testing which differentiate olfactory screening from alternatives like antigen testing and RT-PCR. Because olfaction tests are inexpensive (we estimate $\$ 0.25 /$ test) and uncomplicated, frequent repeated tests can feasibly be carried out by a large fraction of the population. For comparison, to test 50 million people/day (or the entire US population weekly) PCR tests costing $\$ 50$ each would cost $\$ 2.5$ billion per day, $\$ 10$ antigen tests would still cost $\$ 500$ million per day, while an olfactory test would cost $\$ 12.5$ million per day-between $40 \times$ and $200 \times$ less. Moreover, because olfaction can be self-tested, there is no need for the logistics of sample collection and transport, which can further reduce costs. Nevertheless, both RT-PCR and antigen testing have been 
successfully used in screening and surveillance applications, and olfactory screening should be considered as a complement to, not a replacement for, existing successful programs.

A potential advantage of olfactory screening is its ability to be scaled. Paper-based olfactory dysfunction tests can be printed on industrial printers and a single commercial printing facility can produce over 50 million tests daily (D.T., communication). Simple olfaction tests linked to a mobile phone app have already been developed, are FDA registered, and are under consideration for Emergency Use Authorization (EUA) by the U.S. FDA for COVID-19 applications, as of March 2021. In contrast, current PCR testing maximum capacity is approximately 2.1 million tests/day. ${ }^{2}$ Moreover, instant olfactory symptom reporting via a testing app could provide centralized surveillance and early warning of new outbreaks [23]. A facile test may be especially valuable in low and middle income countries where access to complex molecular testing is cost prohibitive.

Our study is subject to a number of limitations. First, our modeling assumed that olfaction tests would not only correctly identify those with olfactory dysfunction, but would remain equally sensitive during regular use. In practice, however, sensitivity could increase or decrease over time as test takers better learned to differentiate the suite of test scents. Second, we assumed that no individual would intentionally fail a testan issue only averted with reflex molecular testing. Finally, we assumed that olfactory dysfunction revealed by a test would lead to isolation, but this assumption is unlikely to be valid for essential workers, or those uninterested in adherence to an isolation protocol. These limitations highlight the need to develop, deploy in longitudinal trials, and refine tests for olfaction that can be mass produced at low cost and self-administered.

There are three additional points to consider in implementing olfactory screening for COVID-19 control. First, when a new community is subject to olfactory screening, there will be an initial surge of individuals identified with olfactory dysfunction both due to undetected COVID-19 infections, and COVID-19independent olfactory dysfunction (anosmia and hyposmia). This will require the ability to handle a reflexive surge in molecular testing during the initial phase. However, even in the absence of follow-up molecular testing, unnecessary quarantines of those without COVID-19 (false positives) should be weighed against blanket non-pharmaceutical interventions such as lockdowns and curfews. A second issue is that increased anosmia and hyposmia in older adults $[36,39,40]$ will mean that olfactory screening will be most effective with younger adults, including college students and school children. Finally, a critical public health issue inherent to any COVID-19 monitoring/surveillance mechanism is that no testing strategy identifies and isolates all the COVID-19 infected individuals, both from false negatives, and from new import of infections into a community. Therefore, any COVID-19 screening regimen must complement and not replace existing viral mitigation mechanisms such as mask wearing and social distancing.

\section{References}

[1] Melissa M Arons, Kelly M Hatfield, Sujan C Reddy, Anne Kimball, Allison James, Jesica R Jacobs, Joanne Taylor, Kevin Spicer, Ana C Bardossy, Lisa P Oakley, et al. Presymptomatic SARS-CoV-2 infections and transmission in a skilled nursing facility. New England Journal of Medicine, 2020.

[2] Desmond Sutton, Karin Fuchs, Mary D'alton, and Dena Goffman. Universal screening for SARSCoV-2 in women admitted for delivery. New England Journal of Medicine, 2020.

\footnotetext{
${ }^{2}$ COVID Tracking Project, as of March 7, 2021; https://covidtracking.com/data.
} 
medRxiv preprint doi: https://doi.org/10.1101/2020.11.30.20241174; this version posted March 15, 2021. The copyright holder for this preprint (which was not certified by peer review) is the author/funder, who has granted medRxiv a license to display the preprint in perpetuity. It is made available under a CC-BY-NC-ND 4.0 International license .

[3] Daniel P Oran and Eric J Topol. Prevalence of asymptomatic SARS-CoV-2 infection: A narrative review. Annals of Internal Medicine, 2020.

[4] Philip Dollard, Isabel Griffin, Andre Berro, Nicole J Cohen, Kimberly Singler, Yoni Haber, Chris de la Motte Hurst, Amber Stolp, Sukhshant Atti, Leslie Hausman, et al. Risk assessment and management of covid-19 among travelers arriving at designated us airports, january 17-september 13, 2020. Morbidity and Mortality Weekly Report, 69(45):1681, 2020.

[5] Michel Bielecki, Giovanni Andrea Gerardo Crameri, Patricia Schlagenhauf, Thomas Werner Buehrer, and Jeremy Werner Deuel. Body temperature screening to identify SARS-CoV-2 infected young adult travellers is ineffective. Travel Medicine and Infectious Disease, 2020.

[6] James L Rudolph, Christopher W Halladay, Malisa Barber, Kevin McCongehy, Vince Mor, Aman Nanda, and Stefan Gravenstein. Temperature in nursing home residents systematically tested for SARS-CoV-2. Journal of the American Medical Directors Association, 2020.

[7] Gary M Vilke, Jesse J Brennan, Alexandrea O Cronin, and Edward M Castillo. Clinical features of patients with COVID-19: is temperature screening useful? The Journal of Emergency Medicine, 2020.

[8] AA Chughtai, Q Wang, TC Dung, and CR Macintyre. The presence of fever in adults with influenza and other viral respiratory infections. Epidemiology \& Infection, 145(1):148-155, 2017.

[9] Daniel C Payne, Sarah E Smith-Jeffcoat, Gosia Nowak, Uzo Chukwuma, Jesse R Geibe, Robert J Hawkins, Jeffrey A Johnson, Natalie J Thornburg, Jarad Schiffer, Zachary Weiner, et al. SARS-CoV-2 infections and serologic responses from a sample of us navy service members-uss theodore roosevelt, april 2020. Morbidity and Mortality Weekly Report, 69(23):714, 2020.

[10] Jerome R Lechien, Carlos M Chiesa-Estomba, Daniele R De Siati, Mihaela Horoi, Serge D Le Bon, Alexandra Rodriguez, Didier Dequanter, Serge Blecic, Fahd El Afia, Lea Distinguin, et al. Olfactory and gustatory dysfunctions as a clinical presentation of mild-to-moderate forms of the coronavirus disease ( COVID-19): a multicenter european study. European Archives of Oto-Rhino-Laryngology, pages $1-11,2020$.

[11] Yury Rusetsky, Irina Meytel, Zhanna Mokoyan, Andrey Fisenko, Anna Babayan, and Ulyana Malyavina. Smell status in children infected with sars-cov-2. The Laryngoscope.

[12] Robert Pellegrino, Ute Walliczek-Dworschak, Gudrun Winter, David Hull, and Thomas Hummel. Investigation of chemosensitivity during and after an acute cold. In International forum of allergy \& rhinology, volume 7, pages 185-191. Wiley Online Library, 2017.

[13] Akosua Adom Agyeman, Ken Lee Chin, Cornelia B Landersdorfer, Danny Liew, and Richard OforiAsenso. Smell and taste dysfunction in patients with COVID-19: A systematic review and metaanalysis. In Mayo Clinic Proceedings. Elsevier, 2020.

[14] Mackenzie E Hannum, Vicente A Ramirez, Sarah J Lipson, Riley D Herriman, Aurora K Toskala, Cailu Lin, Paule V Joseph, and Danielle R Reed. Objective sensory testing methods reveal a higher prevalence of olfactory loss in covid-19-positive patients compared to subjective methods: A systematic review and meta-analysis. Chemical senses, 45(9):865-874, 2020. 
[15] Khang Wen Pang, Jeremy Chee, Somasundaram Subramaniam, and Chew Lip Ng. Frequency and clinical utility of olfactory dysfunction in COVID-19: a systematic review and meta-analysis. Current allergy and asthma reports, 20(12):1-17, 2020.

[16] Carol H Yan, Farhoud Faraji, Divya P Prajapati, Christine E Boone, and Adam S DeConde. Association of chemosensory dysfunction and covid-19 in patients presenting with influenza-like symptoms. In International forum of allergy \& rhinology, volume 10, pages 806-813. Wiley Online Library, 2020.

[17] Andrea Mazzatenta, Giampiero Neri, Damiano D’Ardes, Carlo De Luca, Stefano Marinari, Ettore Porreca, Francesco Cipollone, Jacopo Vecchiet, Chiara Falcicchia, Vincenzo Panichi, et al. Smell and taste in severe covid-19: self-reported vs. testing. Frontiers in Medicine, 7, 2020.

[18] Lucia Iannuzzi, Anna Eugenia Salzo, Gioacchino Angarano, Vincenzo Ostilio Palmieri, Piero Portincasa, Annalisa Saracino, Matteo Gelardi, Michele Dibattista, and Nicola Quaranta. Gaining back what is lost: Recovering the sense of smell in mild to moderate patients after covid-19. Chemical senses, 45(9):875-881, 2020.

[19] Anindya S Bhattacharjee, Samir V Joshi, Shilpa Naik, Shashikala Sangle, and Nixon M Abraham. Quantitative assessment of olfactory dysfunction accurately detects asymptomatic COVID-19 carriers. EClinicalMedicine, page 100575, 2020.

[20] Rafal Butowt and Christopher S von Bartheld. Anosmia in COVID-19: Underlying mechanisms and assessment of an olfactory route to brain infection. The Neuroscientist, page 1073858420956905, 2020.

[21] Yonghyun Lee, Pokkee Min, Seonggu Lee, and Shin-Woo Kim. Prevalence and duration of acute loss of smell or taste in COVID-19 patients. Journal of Korean medical science, 35(18), 2020.

[22] Shima T Moein, Seyed MR Hashemian, Babak Mansourafshar, Ali Khorram-Tousi, Payam Tabarsi, and Richard L Doty. Smell dysfunction: a biomarker for COVID-19. In International forum of allergy \& rhinology. Wiley Online Library, 2020.

[23] Denis Pierron, Veronica Pereda-Loth, Marylou Mantel, Maëlle Moranges, Emmanuelle Bignon, Omar Alva, Julie Kabous, Margit Heiske, Jody Pacalon, Renaud David, et al. Smell and taste changes are early indicators of the covid-19 pandemic and political decision effectiveness. Nature communications, 11(1):1-8, 2020.

[24] Marlene M Speth, Thirza Singer-Cornelius, Michael Oberle, Isabelle Gengler, Steffi J Brockmeier, and Ahmad R Sedaghat. Mood, anxiety and olfactory dysfunction in COVID-19: evidence of central nervous system involvement? The Laryngoscope, 130(11):2520-2525, 2020.

[25] Luigi Angelo Vaira, Giovanna Deiana, Alessandro Giuseppe Fois, Pietro Pirina, Giordano Madeddu, Andrea De Vito, Sergio Babudieri, Marzia Petrocelli, Antonello Serra, Francesco Bussu, et al. Objective evaluation of anosmia and ageusia in COVID-19 patients: Single-center experience on 72 cases. Head \& neck, 42(6):1252-1258, 2020.

[26] Tyler Wagner, FNU Shweta, Karthik Murugadoss, Samir Awasthi, AJ Venkatakrishnan, Sairam Bade, Arjun Puranik, Martin Kang, Brian W Pickering, John C O'Horo, et al. Augmented curation of clinical notes from a massive ehr system reveals symptoms of impending COVID-19 diagnosis. Elife, 9:e58227, 2020. 
medRxiv preprint doi: https://doi.org/10.1101/2020.11.30.20241174; this version posted March 15, 2021. The copyright holder for this preprint (which was not certified by peer review) is the author/funder, who has granted medRxiv a license to display the preprint in perpetuity. It is made available under a CC-BY-NC-ND 4.0 International license .

[27] Alberto Paderno, Davide Mattavelli, Vittorio Rampinelli, Alberto Grammatica, Elena Raffetti, Michele Tomasoni, Tommaso Gualtieri, Stefano Taboni, Silvia Zorzi, Francesca Del Bon, et al. Olfactory and gustatory outcomes in COVID-19: A prospective evaluation in nonhospitalized subjects. Otolaryngology-Head and Neck Surgery, 2020.

[28] Richard C Gerkin, Kathrin Ohla, Maria G Veldhuizen, Paule V Joseph, Christine E Kelly, Alyssa J Bakke, Kimberley E Steele, Michael C Farruggia, Robert Pellegrino, Marta Y Pepino, et al. Recent smell loss is the best predictor of covid-19 among individuals with recent respiratory symptoms. Chemical Senses, 46, 2021.

[29] Daniel B Larremore, Bryan Wilder, Evan Lester, Soraya Shehata, James M Burke, James A Hay, Milind Tambe, Michael J Mina, and Roy Parker. Test sensitivity is secondary to frequency and turnaround time for COVID-19 surveillance. MedRxiv, 2020.

[30] Michael J Mina, Roy Parker, and Daniel B Larremore. Rethinking COVID-19 test sensitivity—a strategy for containment. New England Journal of Medicine, 2020.

[31] A David Paltiel, Amy Zheng, and Rochelle P Walensky. Assessment of SARS-CoV-2 screening strategies to permit the safe reopening of college campuses in the united states. JAMA network open, 3(7):e2016818-e2016818, 2020.

[32] Stephen M Kissler, Joseph R Fauver, Christina Mack, Caroline Tai, Kristin Y Shiue, Chaney Kalinich, Sarah Jednak, Isabel Ott, Chantal Vogels, Jay Wohlgemuth, et al. Viral dynamics of SARS-CoV-2 infection and the predictive value of repeat testing. medRxiv, 2020.

[33] Muge Cevik, Matthew Tate, Oliver Lloyd, Alberto Enrico Maraolo, Jenna Schafers, and Antonia Ho. SARS-CoV-2, sars-cov-1 and mers-cov viral load dynamics, duration of viral shedding and infectiousness: a living systematic review and meta-analysis. medRxiv, 2020.

[34] Amber M Smith and Alan S Perelson. Influenza A virus infection kinetics: quantitative data and models. Wiley Interdisciplinary Reviews: Systems Biology and Medicine, 3(4):429-445, 2011.

[35] Mathilde Richard, Adinda Kok, Dennis de Meulder, Theo M Bestebroer, Mart M Lamers, Nisreen MA Okba, Martje Fentener van Vlissingen, Barry Rockx, Bart L Haagmans, Marion PG Koopmans, et al. SARS-CoV-2 is transmitted via contact and via the air between ferrets. bioRxiv, 2020.

[36] Jingpu Yang and Jayant M Pinto. The epidemiology of olfactory disorders. Current otorhinolaryngology reports, 4(2):130-141, 2016.

[37] Heike Rebholz, Ralf J Braun, Dennis Ladage, Wolfgang Knoll, Christoph Kleber, and Achim W Hassel. Loss of olfactory function - early indicator for covid-19, other viral infections and neurodegenerative disorders. Frontiers in Neurology, 11, 2020.

[38] Mohammad Waheed El-Anwar, Saad Elzayat, and Yasser Ahmed Fouad. Ent manifestation in covid-19 patients. Auris Nasus Larynx, 47(4):559-564, 2020.

[39] Sanne Boesveldt, Elbrich M Postma, Duncan Boak, Antje Welge-Luessen, Veronika Schöpf, Joel D Mainland, Jeffrey Martens, John Ngai, and Valerie B Duffy. Anosmia-a clinical review. Chemical senses, 42(7):513-523, 2017. 
medRxiv preprint doi: https://doi.org/10.1101/2020.11.30.20241174; this version posted March 15, 2021. The copyright holder for this preprint (which was not certified by peer review) is the author/funder, who has granted medRxiv a license to display the preprint in perpetuity.

It is made available under a CC-BY-NC-ND 4.0 International license .

[40] Andreas Hinz, Tobias Luck, Steffi G Riedel-Heller, Philipp Y Herzberg, Claudia Rolffs, Kerstin Wirkner, and Christoph Engel. Olfactory dysfunction: properties of the sniffin'sticks screening 12 test and associations with quality of life. European Archives of Oto-Rhino-Laryngology, 276(2):389$395,2019$.

[41] Kendra Quicke, Emily Gallichote, Nicole Sexton, Michael Young, Ashley Janich, Gregory Gahm, Elizabeth J Carlton, Nicole Ehrhart, and Gregory D Ebel. Longitudinal surveillance for SARS-CoV-2 RNA among asymptomatic staff in five colorado skilled nursing facilities: Epidemiologic, virologic and sequence analysis. medRxiv, 2020.

[42] Roman Wölfel, Victor M Corman, Wolfgang Guggemos, Michael Seilmaier, Sabine Zange, Marcel A Müller, Daniela Niemeyer, Terry C Jones, Patrick Vollmar, Camilla Rothe, et al. Virological assessment of hospitalized patients with COVID-2019. Nature, 581(7809):465-469, 2020.

[43] Bernard La Scola, Marion Le Bideau, Julien Andreani, Van Thuan Hoang, Clio Grimaldier, Philippe Colson, Philippe Gautret, and Didier Raoult. Viral RNA load as determined by cell culture as a management tool for discharge of SARS-CoV-2 patients from infectious disease wards. European Journal of Clinical Microbiology \& Infectious Diseases, 39(6):1059, 2020.

[44] Genay Pilarowski, Carina Marquez, Luis Rubio, James Peng, Jackie Martinez, Douglas Black, Gabriel Chamie, Diane Jones, Jon Jacobo, Valerie Tulier-Laiwa, et al. Field performance and public health response using the binaxnow tm rapid sars-cov-2 antigen detection assay during community-based testing. Clinical Infectious Diseases, 2020.

[45] Luigi Angelo Vaira, Giovanni Salzano, Marzia Petrocelli, Giovanna Deiana, Francesco Antonio Salzano, and Giacomo De Riu. Validation of a self-administered olfactory and gustatory test for the remotely evaluation of covid-19 patients in home quarantine. Head \& neck, 42(7):1570-1576, 2020.

[46] Daniel Hornuss, Berit Lange, Nils Schroeter, Siegbert Rieg, Winfried V Kern, and Dirk Wagner. Anosmia in covid-19 patients. Clinical Microbiology and Infection, 2020.

[47] Luigi Angelo Vaira, Claire Hopkins, Giovanni Salzano, Marzia Petrocelli, Andrea Melis, Marco Cucurullo, Mario Ferrari, Laura Gagliardini, Carlotta Pipolo, Giovanna Deiana, et al. Olfactory and gustatory function impairment in covid-19 patients: Italian objective multicenter-study. Head \& neck, 42(7):1560-1569, 2020.

\section{Acknowledgements}

The authors thank Yonatan Grad and A. Marm Kilpatrick for valuable feedback. This work was supported by the Howard Hughes Medical Institute (Roy Parker).

\section{Author Contributions}

D.B.L., D.T., and R.P. conceived and designed the study. D.B.L. performed the computational modeling. D.B.L., D.T., and R.P. wrote the manuscript.

\section{Data Availability}

All data needed to evaluate the conclusions in the paper are present in the paper and/or the Supplementary Materials, and open-source code is available at https://github.com/LarremoreLab/covid_olfactory_dysfunction. 
medRxiv preprint doi: https://doi.org/10.1101/2020.11.30.20241174; this version posted March 15, 2021. The copyright holder for this preprint (which was not certified by peer review) is the author/funder, who has granted medRxiv a license to display the preprint in perpetuity. It is made available under a CC-BY-NC-ND 4.0 International license.

\section{Competing Interests}

D.B.L. is a member of the scientific advisory board of Darwin BioSciences. D.T. is a founder of an olfactory test company u-Smell-it LLC and has related pending patents. 


\section{Materials and Methods}

\section{Individual infection model}

Each individual infection consists of four key elements: (1) a viral load trajectory which charts the measurable concentration of virus over the course of infection, (2) the presence of symptoms so noticeable that they cause a change in behavior, i.e. symptom-driven self-isolation, (3) the presence of symptoms identifiable by a screening test, such as olfactory dysfunction or low fever, and (4) an infectiousness trajectory, which is related to both viral load and behavior. Each of these components is described in detail below.

When a positive screening test-virological or symptom-based-provides a positive test result during the individual's infectious window, prior to any self-isolation, that person is assumed to isolate for the remainder of the infection. Thus, depending on the individual's viral load, infectiousness, timing of possible test results, and timing of possible self-isolation, a screening test may or may not cause an individual's isolation and concomitant decrease in circulating infectiousness.

Viral Load Trajectories. Viral loads were drawn from a simple, previously published viral kinetics model which captures four key aspects of a SARS-CoV-2 infection [29]: (a) a variable latent period, (b) a rapid proliferation phase from the lower limit of PCR detectability to a peak viral load, (c) a slower clearance phase, and (d) prolonged clearance for symptomatic infections vs asymptomatic infections. These dynamics were based on a growing body of literature that includes longitudinal repeated PCR sampling of symptomatic and asymptomatic individuals, in both prospective and non-prospective contexts. The detailed studies on which the viral load was based were previously reviewed in [29], but have since been further supported through a prospective longitudinal study [32] which further refined the proliferation phase and differential clearance rates based on symptoms.

To summarize the model of Ref. [29], with slight modifications to reflect knowledge gained from Ref. [32], $\log _{10}$ viral loads were approximated by a continuous piecewise linear "hinge" function, specified uniquely with three control points: $\left(t_{0}, 3\right),\left(t_{\text {peak }}, V_{\text {peak }}\right),\left(t_{f}, 6\right)$. The first point represents the time at which an individual's viral load first crosses $10^{3}$, and becomes detectable via PCR, with $t_{\mathrm{PRCR}} \sim$ unif $[2.5,3.5]$, measured in days since exposure. The second point represents the peak viral load. Peak height was drawn $V_{\text {peak }} \sim$ unif[7,11], and peak timing was drawn with respect to the start of the proliferation phase, $t_{\text {peak }}-t_{\mathrm{PCR}} \sim 0.5+\operatorname{gamma}(2.5)$ with a maximum of 4 . The third point represents the time at which an individual's viral load crosses beneath the $10^{6}$ threshold, at which point viral loads no longer cause active cultures in laboratory experiments [33, 41-43]. For asymptomatic infections, this point was drawn with respect to peak timing, $t_{f}-t_{\text {peak }} \sim$ unif $[4,8]$. For overtly symptomatic infections, a symptom onset time was first drawn with respect to peak timing, $t_{\text {symptoms }}-t_{\text {peak }} \sim$ unif[0,2], and then the third control point was drawn with respect to symptom onset, $t_{f}-t_{\text {symptoms }} \sim$ unif $[4,8]$. Thus, overtly symptomatic trajectories are systematically longer, in both duration of infectiousness and duration of viral shedding [32]. In simulations, each viral load's parameters were drawn independently of others, and the continuous function described here was evaluated at 21 integer time points, representing a three week span of viral load values.

Overt symptoms causing self-isolation. For individuals with overtly symptomatic infections (see description of Viral Load Trajectories), symptom onset at $t_{\text {symptoms }}$ caused self-isolation on the following day. Overt symptoms were assumed to be present in $35 \%$ of individuals, and were assumed to be present independently of olfactory dysfunction and low-grade fever, which are considered separately because they typically do not lead to behavior change. 
Analytical sensitivity of RT-PCR and antigen testing. We assume an analytical sensitivity of RT-PCR of $10^{3}$ RNA copies/ml, consistent with typical performance. We assume an analytical sensitivity of antigen tests equivalent to $10^{6} \mathrm{RNA}$ copies $/ \mathrm{ml}$, consistent with independent field evaluations of the Abbott BinaxNOW test [44]. When viral loads exceeded their respective limits of detection, we assume that these molecular tests correctly identify infected individuals.

Symptoms identifiable by screening. Symptoms such as olfactory dysfunction or low-grade fever were modeled using three variables which controlled the prevalence $\theta$, onset time $t_{\text {onset }}$, and duration $d$ of symptoms. Onset times were chosen relative to detectability by PCR, such that $t_{\text {onset }}=t_{\mathrm{PCR}}+k$, with $k=$ $-2,-1, \ldots, 3$ evaluated in the text. Note that the prevalence parameter $\theta$ incorporates both the presence and the detectability of symptoms. As noted above, the prevalence of identifiable symptoms via this mechanism was considered independently of the overt symptoms that lead to self-isolation.

Infectiousness. Infectiousness $F$ was assumed to be directly related to viral load $V$ in that each individual's relative infectiousness was proportional to the $\log _{10}$ of viral load's excess beyond $10^{6}$, i.e. $F \propto \log _{10}(V)-6$. For individuals in isolation, either following a positive screening test or following the appearance of overt symptoms (at $t_{\text {symptoms }}$ ), infectiousness was set to zero. In all simulations, the value of the proportionality constant implied by the infectiousness function was chosen to achieve the targeted value of $R_{0}$ for that simulation, and confirmed via simulation as described below.

\section{One-shot, Two-shot, and Repeated Screening}

For an individual with viral load trajectory $V$ and infectiousness $F$ over the simulated 21 days of infection, proliferation, and clearance, testing was implemented on a specified day or on a schedule, as follows. First, each individual's viral load $V$ was drawn as described above, with $35 \%$ of individuals receiving an overtly symptomatic trajectory and $65 \%$ receiving an overtly asymptomatic trajectory. Each individual was also assigned screenable symptoms with probability $\theta$, lasting from day $t_{\text {onset }}$ through $t_{\text {onset }}+d$, and no screenable symptoms otherwise.

One-shot and two-shot screening. For disease transmission dynamics scenarios, on the day of one-shot screening, each individual was evaluated to determine whether they would receive a positive test result that day (symptom screening) or the next day (virological screening). Thus, the timing of each individual's viral load, symptom status, and infection status were determined by the dynamics of the simulation, described below. For non-dynamic scenarios, a statistical sample of 10,000 infected individuals were considered such that the timing of the screening test was equiprobable on each day of infection, with symptom screening and virological results returned on the same day and next day, respectively. Olfactory dysfunction tests were assumed to have a specificity of either 0.8 or 0.96 , to explore the impact of vary rates of COVID-19independent olfactory dysfuction.

In two-shot screening scenarios, individuals with positive symptom screening tests were referred to a reflex point-of-care rapid diagnostic test with an assumed limit of detection $L$. If $V(t)>L$ on that day, they received a positive result and isolated, but otherwise, they received a negative result and were allowed to enter the imagined event. Rapid diagnostic tests were assumed to have a specificity of 0.025 . False positives by olfactory dysfunction test and by rapid diagnostic test were assumed to be statistically independent of each other, such that the specificity of the two-shot screening was 0.001 .

Repeated screening. Based on a schedule of testing each person every $D$ days, if an individual happened to be tested by a symptom screening test on a day when their symptoms were present, their positive result would 
cause them isolate that day, without delay. Similarly, if an individual happened to be tested by a virological test on a day when their viral load exceeded the limit of detection of the test $(V(t)>L)$, their positive result would cause them to isolate, but with a one day delay in virological test results. Each person was deterministically tested exactly every $D$ days, but testing days were drawn uniformly at random such that not all individuals were tested on the same day. Values of $D$ were 1,3 or 7 . In repeated screening scenarios, olfactory dysfunction tests were assumed to have a specificity of 1 , reflecting a steady-state assumption that individuals would rapidly determine that their olfactory dysfunction was not COVID-19-related.

\section{Disease Transmission Model}

A fully-mixed model of $N=20,000$ individuals with all-to-all contact structure, zero initial infections, and a constant $1 / N$ per-person probability of becoming infected from an external source was used to simulate SARS-CoV-2 dynamics, based on a typical compartmental framework, as described in Ref. [29] but with modifications for symptom screening. This models tracked discrete individuals who were Susceptible $(S)$, Infected $(I)$, Recovered $(R)$, Isolated $(Q)$, and Self-Isolated $(S Q)$ at each discrete one day timestep. Upon becoming infected $(S \rightarrow I)$, a viral load trajectory $V(t)$ was drawn which included a latent period, growth, and decay as described above. For those chosen to have non-overt symptoms, the timing and duration were fixed, according the choice of parameters and the $t_{\mathrm{PCR}}$ of the viral load. Thus, each day, an individual's viral load trajectory was used to determine whether their diagnostic test would be positive if administered, as well as their infectiousness to susceptible individuals; The timing, duration, and prevalence of symptoms was used similarly to determine whether a screening test would be positive if administered.

Participation in testing. $20 \%$ of individuals were, in some simulations, selected to refuse testing. Testing refusal—or its complement, participation-was determined at random using a specified refusal rate, at the initialization of each simulation, and was unchanged for the duration of each simulation. In all cases except for the one-shot and two-shot screening scenarios, we considered an $80 \%$ participation rate (i.e. at $20 \%$ refusal rate).

Some individuals who are interested in testing (i.e. they do not refuse a testing regimen) may nevertheless be unable to effectively participate due to existing full or partial loss of olfaction [36]. To conservatively model the impact of olfactory screening, particularly among older adults, we considered scenarios where either $5 \%$ of individuals or $20 \%$ of individuals, as noted throughout the text, had COVID-19-independent olfactory loss which rendered them unable to participate in testing programs.

Taken together, these two participation rates mean that for repetitive screening scenarios and simulations we assumed that only $80 \% \times 80 \%=64 \%$ of the population could effectively participate in a screening regimen. In one-shot and two-shot screening scenarios, only the inability to participate was considered, such that the specificity of the olfactory screening was either $95 \%$ or $80 \%$.

Isolation due to symptom screening or virological testing. On the specified day(s) of testing, each infected person was evaluated to determine whether their viral load $(V(t)>L$ in the case of a virological test) or symptoms (presence/absence for that individual on that day, in the case of a symptom screening test) would cause a positive test result. Positive results caused isolation $(I \rightarrow Q)$ with no delay or with a one day delay for symptom screening or virological tests, respectively.

Self-isolation and recovery. $35 \%$ of individuals self-isolate on the day of symptom onset, which occurs 0 to 3 days after peak viral load (see above), to mimic overt symptom-driven isolation $(I \rightarrow S Q)$. Thus, presymptomatic individuals were isolated prior to symptom onset only if they received positive test results. 
When an individual's viral load dropped below $10^{3}$, that individual recovered $(I, Q, S Q \rightarrow R)$.

Transmission, Population Structure, and Mixing Patterns. Simulations were initialized with all individuals susceptible, $S=N$. Each individual was initially chosen to either participate in testing or refused testing, as described above, independently with a probability specified per-simulation. Each individual was chosen to be overtly symptomatic independently with probability 0.35 . Both participation/refusal and overt symptoms were assumed to be persistent through the simulation, per person. If repeated testing was to be performed, each individual's first test day (e.g. the day of the week that their weekly test would occur) was chosen uniformly at random between 1 and $D$. Relative infectiousness was scaled up or down to achieve the specified $R_{0}$ in the absence of any testing policy, but inclusive of any assumed self-isolation of overt symptomatics.

In each timestep, those individuals who were marked for testing that day were tested. Individuals receiving a positive test result that day, after delay of virological test results, were isolated, $I \rightarrow Q$. Overtly symptomatic individuals whose viral load had declined relative to the previous day were self-isolated, $I \rightarrow S Q$. Next, each susceptible individual was spontaneously (externally) infected independently with probability $1 / N, S \rightarrow I$. Then, all infected individuals contacted all susceptible individuals, with the probability of transmission based on that day's viral load $V(t)$ for each person infectiousness function described above, $S \rightarrow I$.

To conclude each time step, individuals' viral loads and symptoms were advanced to the next time step, with those whose infectious period had completely passed moved to recovery, $I, Q, S Q \rightarrow R$.

Ongoing screening vs outbreak mitigation scenarios. In ongoing screening scenarios, simulations with $R_{0}=1.5$ and the constant rate of external infection were conducted with screening beginning, as described above, starting on the first timestep. In outbreak mitigation scenarios, simulations were identical except that no screening was performed until disease prevalence in that time step reached $2 \%$ of the population (400 individuals).

\section{Calibration to achieve targeted $R_{0}$ and estimation of $R$}

As a consistency check, each simulation's $R_{0}$ was confirmed to ensure that simulations were properly calibrated to their intended values. Note that to vary $R_{0}$, the proportionality constant in the function that maps viral load to infectiousness need only be adjusted up or down. In a typical SEIR model, this would correspond to changing the infectiousness parameter which governs the rate at which $I$-to- $S$ contacts cause new infections $\beta$.

For the fully-mixed model, the value of $R_{0}$ was numerically estimated by running single-generation simulations in which a 50 infected individual were placed in a population of $N-50$ others. The number of secondary infections from those initially infected was recorded and used to directly estimate $R_{0}$.

Estimations of $R$ proceeded exactly as estimations of $R_{0}$ for both models, except with interventions applied to the the viral loads, symptoms, and therefore the dynamics. 
medRxiv preprint doi: https://doi.org/10.1101/2020.11.30.20241174; this version posted March 15, 2021. The copyright holder for this preprint (which was not certified by peer review) is the author/funder, who has granted medRxiv a license to display the preprint in perpetuity.

It is made available under a CC-BY-NC-ND 4.0 International license.

\section{Supplemental Figures and Tables}

\section{Contents}

- Supplementary Figures S1-S5

- Supplementary Table S1

- Supplementary Text 


\begin{tabular}{|c|c|c|c|c|c|}
\hline Reference Author & $\begin{array}{l}\text { Objective } \\
\text { Olfactory } \\
\text { Test Type }\end{array}$ & Country & $\mathbf{N}$ & $\begin{array}{c}\text { Olfactory } \\
\text { Dysfunction }^{b}\end{array}$ & Comments \\
\hline Moein et al. [22] & $\begin{array}{l}\text { UPSIT } \\
\text { (40 odorants) }\end{array}$ & Iran & 60 & $98 \%[91,100]$ & $\begin{array}{l}\text { Adults, moderate } \\
\text { and severe }\end{array}$ \\
\hline Vaira et al. [45] & CCCRC & Italy & 72 & $61 \%[65,74]$ & Adults, symptomatic \\
\hline Hornuss et al. [46] & Sniffin' Sticks & Germany & 45 & $84 \%[71,92]$ & Adults, symptomatic \\
\hline Lechien et al. [10] & Sniffin' Sticks & Belgium & 86 & $84 \%[35,67]$ & Adults, symptomatic \\
\hline Vaira et al. [25] & CCCRC & Italy & 33 & $52 \%[35,67]$ & Adults, symptomatic \\
\hline Vaira et al. [47] & CCCRC & Italy & 345 & $70 \%[65,74]$ & Adults, symptomatic \\
\hline Bhattacharjee et al. [19] & Custom & India & $70^{a}$ & $82 \%$ [N.D.] & $\begin{array}{l}\text { Adults, asymptomatic. } \\
15 \% \text { self-reported LOS }\end{array}$ \\
\hline Rusetsky et al. [11] & Sniffin' Sticks SIT & Russia & 79 & $86 \%$ [N.D.] & $\begin{array}{l}\text { Children. } 48 \% \text { recovered } \\
\text { from LOS after } 5 \text { days. }\end{array}$ \\
\hline
\end{tabular}

Table S1: Frequency of olfactory dysfunction (OD) in COVID-19 subjects when monitored with a quantitative olfactory test device. For all studies, PCR for SARS CoV-2 was used as the "gold standard" reference test. Data are expanded and modified from [13] and [14]. SIT, Smell Identification Test; CCRC, Connecticut Chemosensory Clinical Research Center; LOS, Loss of Smell; N.D., not determined.

a 37 healthy and 33 asymptomatic COVID-19 positive patients.

${ }^{b}$ Percentages represent central estimates, with 95\% confidence intervals in brackets. Across studies, most tests were performed a single point in the clinical course. Percentage of PCR-positive COVID-19 cases with confirmed OD are therefore estimates of the sensitivity of OD as a predictor of OD. True prevalence of the symptom may therefore vary if windows of PCR positive and OD are imperfectly overlapping. 

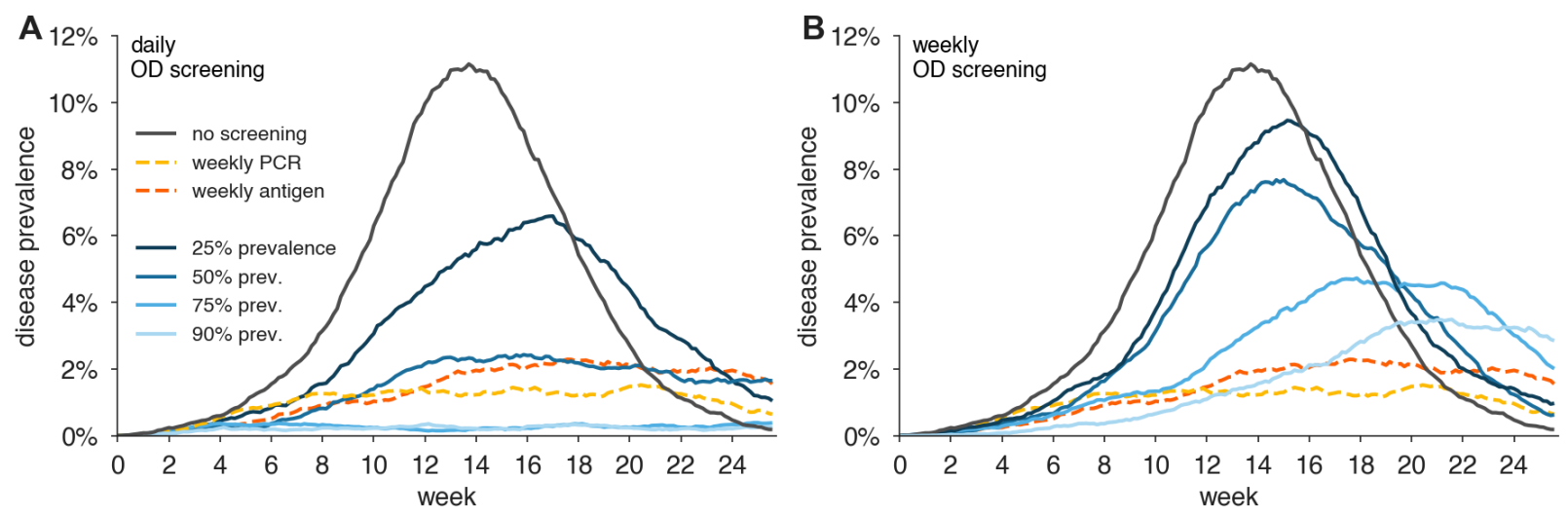

Figure S1: Impact of olfactory dysfunction prevalence on its effectiveness to limit viral spread. The figure shows examples of viral spread in fully-mixed community of 20,000 individuals performing olfactory dysfunction (OD) screening daily (A), or weekly (B). No mitigation (black), Prevalence of symptom shown are: $25 \%$ (dark blue), 50\% (medium blue), 75\% (light blue), 90\% (lightest blue). For comparison, weekly RT-PCR testing with a one day turnaround is shown. In this analysis, olfactory dysfunction is modeled to last 7 days, and begin two days after viral levels reach 1000 virions $/ \mathrm{ml}$. We consider $80 \%$ participation in testing and that $20 \%$ of individuals would suffer COVID-19-independent olfactory dysfunction which would exclude them from effective testing. 

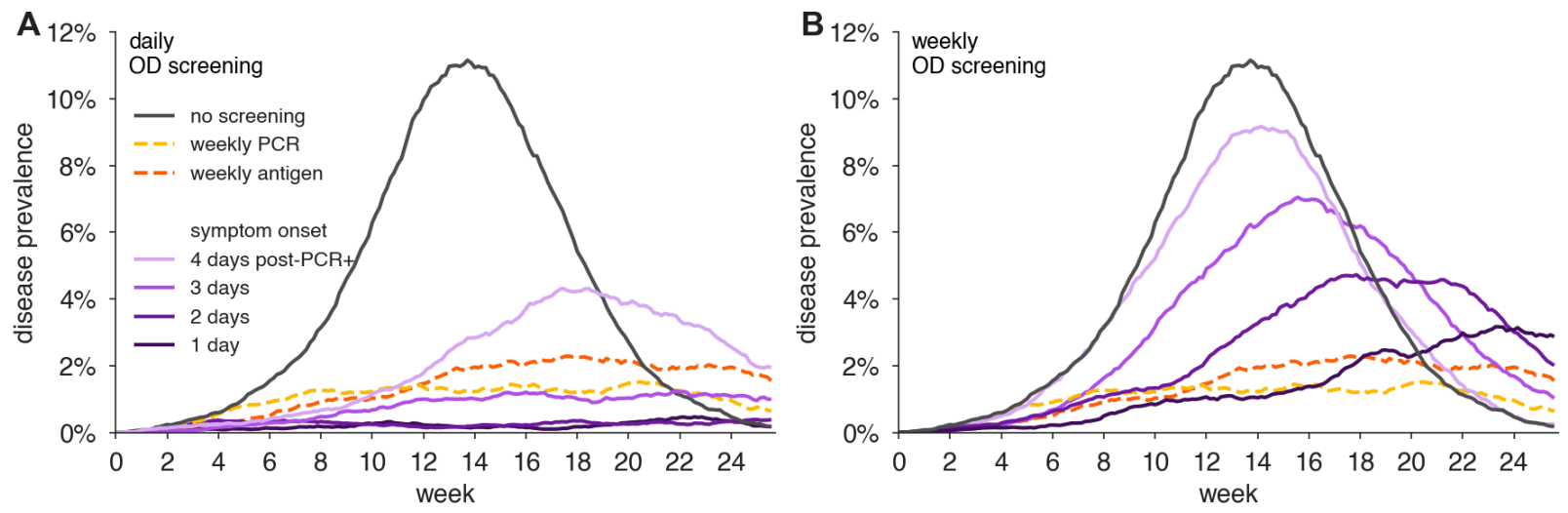

Figure S2: Impact of the timing of olfactory dysfunction onset on its effectiveness to limit viral spread. Example of viral spread in fully-mixed community of 20,000 individuals performing olfactory dysfunction (OD) screening daily (A) or weekly (B). olfactory dysfunction is modeled to be present in $75 \%$ of infected individuals, and to last 7 days. We consider $80 \%$ participation in testing and that $20 \%$ of individuals would suffer COVID-19-independent olfactory dysfunction which would exclude them from effective testing. Timing of olfactory dysfunction is varied from one to four days after virions levels reaching 1000 virions/ml (purple shaded lines as indicated). No mitigation is shown as black line. For comparison, weekly RT-PCR testing with a one day turnaround is shown.
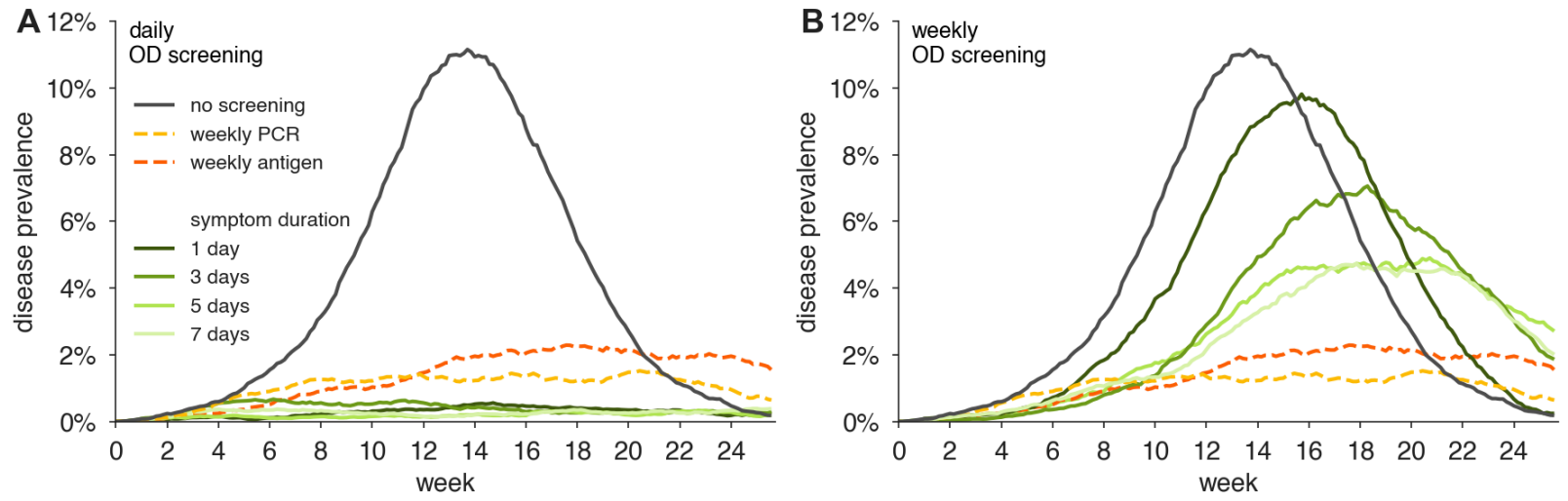

Figure S3: Impact of duration of olfactory dysfunction on its effectiveness to limit viral spread. Examples of viral spread in fully-mixed community of 20,000 individuals performing olfactory dysfunction (OD) screening daily (A) or weekly (B). Olfactory dysfunction is modeled to be present in $75 \%$ of infected individuals, and to begin two days after virion levels reach 1000 virions $/ \mathrm{ml}$. We consider $80 \%$ participation in testing and that $20 \%$ of individuals would suffer COVID-19-independent olfactory dysfunction which would exclude them from effective testing. Duration of olfactory dysfunction is varied from 7 days (lightest green), 5 days (light green), 3 days (green), to 1 day (dark green). No mitigation is shown as black line. For comparison, weekly RT-PCR testing with a one day turnaround is shown. 

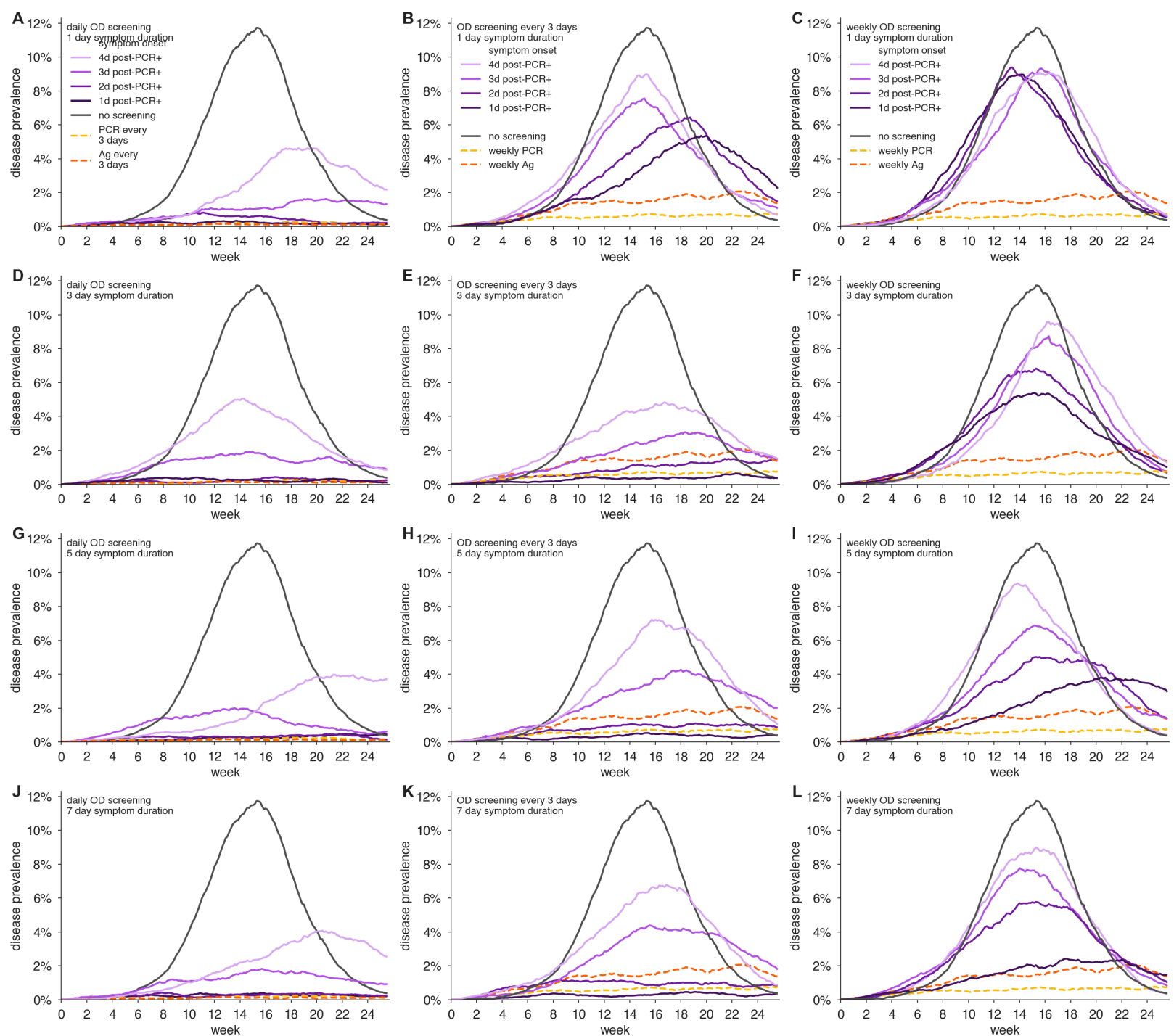

Figure S4: Impact of varying duration and onset time of olfactory dysfunction on its effectiveness to limit viral spread. Examples of viral spread in fully-mixed community of 20,000 individuals performing olfactory dysfunction (OD) screening daily for symptoms lasting (A, B, C) one day, (D, E, F) three days, (G, H, I) five days, and (J, $\mathrm{K}, \mathrm{L}$ ) seven days, screening every day (left column), every three days (middle column) and weekly (right column). olfactory dysfunction is modeled to be present in $75 \%$ of infected individuals, and to begin after 1, 2, 3, or 4 days from when virion levels reach 1000 virions $/ \mathrm{ml}$, indicated by varying shades of purple (see legend). We consider $80 \%$ participation in testing and that $20 \%$ of individuals would suffer COVID-19-independent olfactory dysfunction which would exclude them from effective testing. No mitigation is shown as black line. For comparison, RT-PCR testing with a one day turnaround and antigen testing are shown with testing every three days (left column) or weekly (middle and right columns). 

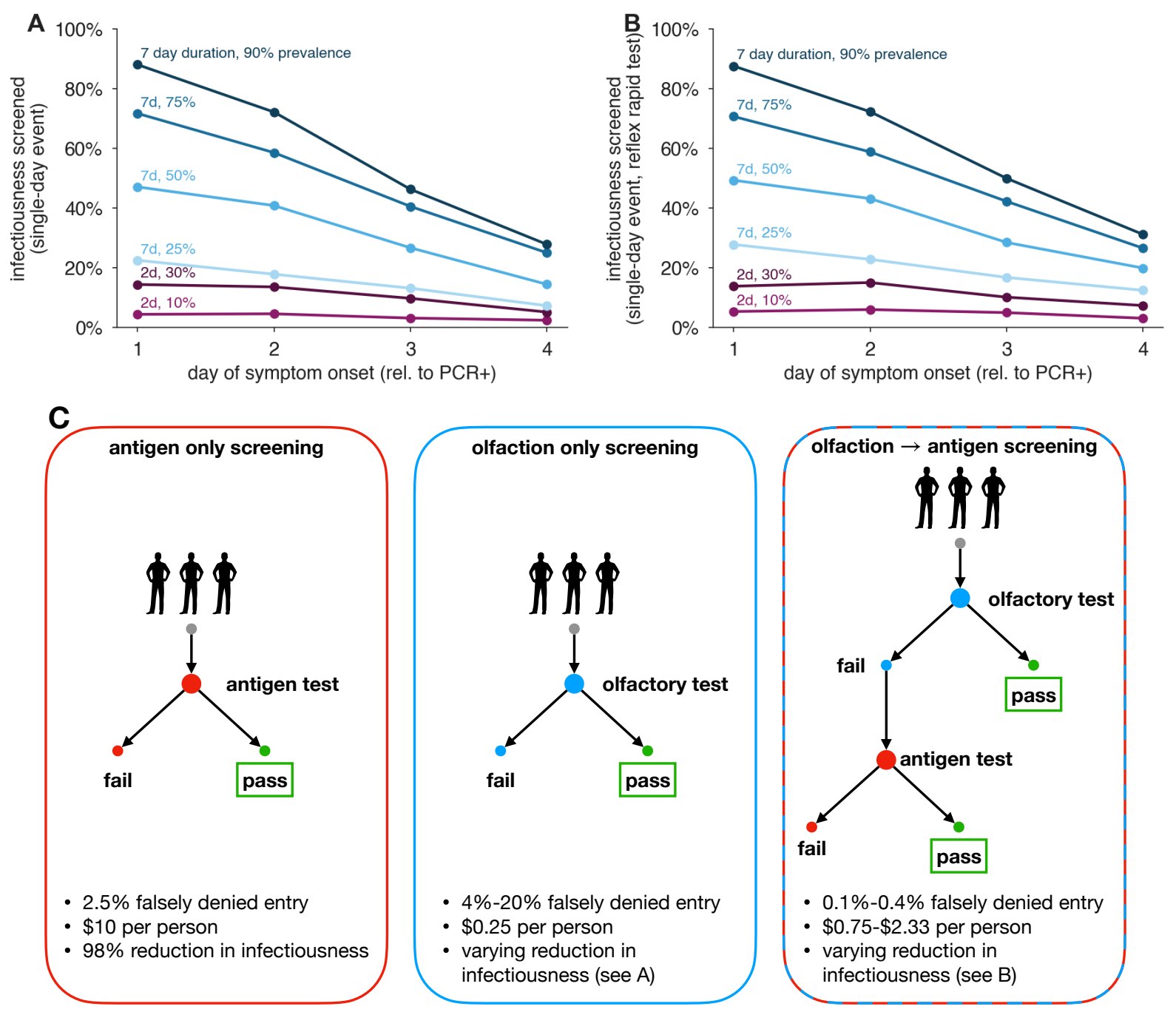

Figure S5: Olfactory dysfunction and antigen testing for point-of-entry screening. (A,B) Lines show infectiousness screened for various combinations of symptom prevalence, duration, and onset, without (A) and with (B) reflex testing of those who fail the olfactory test with a rapid antigen test. Blue lines model olfactory dysfunction: $90 \%$ (dark blue), 75\% (medium blue), 50\% (light blue). Red lines model fever: 30\% (dark red), 10\% (light red). d, duration of symptom in days. (C) Diagram shows the impacts of antigen-based, olfaction-based, and olfaction+antigen screening as point of entry testing. The combined olfaction+antigen approach results in a low-cost, highly effective, and high specificity point-of-entry screening. 


\section{Supplemental Text}

\section{S1 Predicting the impact of repeated population screening testing on $R$}

The impact of repeated population screening on the reproductive number can be estimated by considering the ratio of population infectiousness with a screening regimen to population infectiousness with no screening. However, note that the impact of a population screening policy may depend on two additional factors.

First, not all individuals may wish to participate in a testing program. Let the fraction of individuals who participate be given by $\phi$.

Second, a virological test (e.g. RT-PCR) may produce a false negative result unrelated to its limit of detection-for instance due to an improperly collected sample. Let se be the test sensitivity, in the particular sense of the probability of correctly diagnosing an individual as positive when that person's viral load should, in principle, have provided a sufficiently high RNA concentration to be detectable.

Let $f_{0}$ be the total infectiousness removed with no testing policy, i.e. due to symptom-driven self isolation. Let $f_{\text {test }}(s e)$ be the fraction of total infectiousness removed with a chosen testing policy, inclusive of symptom-driven self isolation, as well as the test sensitivity $s e$ introduced above. The quantity $f_{\text {test }}$ may be computed for any screening program, including virological testing or symptom screening.

Both $f_{0}$ and $f_{\text {test }}(s e)$ can be estimated rapidly via Monte Carlo by drawing trajectories and applying a population screening regimen to them in which a fraction 1 - se positive tests are discarded uniformly at random. In the main text, we found that estimating these values using 10,000 randomly drawn trajectories was sufficient to produce stable estimates.

Under the assumption of statistical independence between an individual's participation or refusal, viral load or olfactory dysfunction status, and $s e$, we can approximate the reproductive number as

$$
R \approx\left[\phi \frac{1-f_{\text {test }}(s e)}{1-f_{0}}+1-\phi\right] R_{0}
$$

which simply expresses a weighted combination of removed infectiousness via screening regimen participation and no test. Intuitively, note that if there is complete refusal to participate $(\phi=0)$ or an entirely ineffective test $\left(f_{\text {test }}(s e)=f_{0}\right)$, then $R \approx R_{0}$, as expected. 